\title{
Quantification in frame semantics with binders and nominals of hybrid logic
}

\author{
Laura Kallmeyer ${ }^{1}$, Rainer Osswald ${ }^{1}$, and Sylvain Pogodalla ${ }^{1,2}$ \\ ${ }^{1}$ Heinrich Heine Universität, Düsseldorf, Germany \\ 2 INRIA, Villers-lès-Nancy, France \\ Université de Lorraine, LORIA, Vandœuvre-lès-Nancy, France \\ CNRS, LORIA, Vandœuvre-lès-Nancy, France
}

\begin{abstract}
This paper aims to integrate logical operators into frame-based semantics. Frames are semantic graphs that allow lexical meaning to be captured in a fine-grained way but that do not come with a natural way to integrate logical operators such as quantifiers. The approach we propose stems from the observation that modal logic is a powerful tool for describing relational structures, including frames. We use its hybrid logic extension in order to incorporate quantification and thereby allow for inference and reasoning. We integrate our approach into a type theoretic compositional semantics, formulated within Abstract Categorial Grammars. We also show how the key ingredients of hybrid logic, nominals and binders, can be used to model semantic coercion, such as the one induced by the begin predicate. In order to illustrate the effectiveness of the proposed syntax-semantics interface, all the examples can be run and tested with the Abstract Categorial Grammar development toolkit.

1

FRAMES AND LEXIGAL SEMANTIGS

Frames emerged as a representation format of conceptual and lexical knowledge (Fillmore 1977; Barsalou 1992; Löbner 2014a). They

"This work was supported by the INRIA sabbatical program and by the CRC 991 "The Structure of Representations in Language, Cognition, and Science" funded by the German Research Foundation (DFG).
\end{abstract}

Keywords: Frame semantics, quantification, hybrid logic, Abstract Categorial Grammar 
are commonly presented as semantic graphs with labelled nodes and edges, such as the one in Figure 1, where nodes correspond to entities (individuals, events, ...) and edges correspond to (functional or nonfunctional) relations between these entities. In Figure 1 all relations except part-of are meant to be functional.

Figure 1:

Frame for the meaning of the man walked to the house (adapted from Kallmeyer and Osswald 2013)

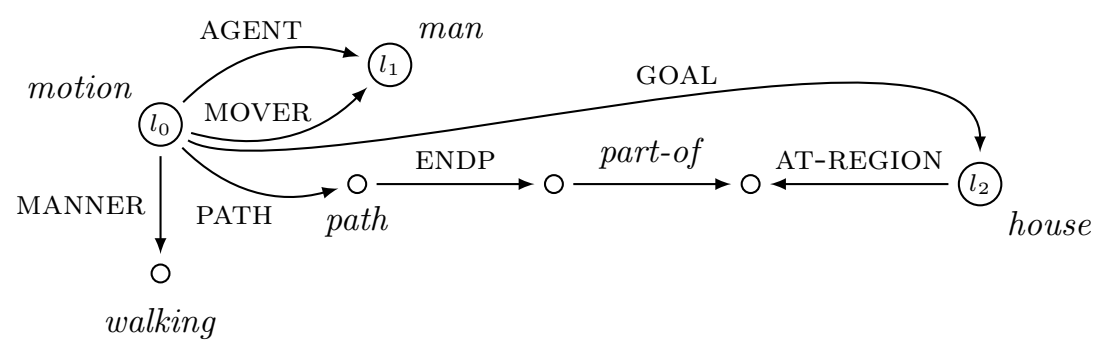

Structuring the knowledge as frames offers a fine-grained and systematic decomposition of meaning. This conception of frames is however not to be confused with the somewhat simpler FrameNet frames, although the former can help to capture the structural relations of the latter (see Osswald and Van Valin 2014).

Frames can be formalized as extended typed feature structures (Petersen 2007; Kallmeyer and Osswald 2013) and specified as models of a suitable logical language, the labelled attribute-value description ( LAVD) language. Such a language allows for the composition of lexical frames on the sentential level by means of an explicit syntax-semantics interface (Kallmeyer and Osswald 2013).

1.1

Logical representation of feature structures

The syntax-semantics interface of (Kallmeyer and Osswald 2013) relies on a formal representation of semantic frames as base-labelled feature structure with types and relations. This definition extends the standard definition of feature structures in two respects. First, in addition to features, proper relations between nodes can be expressed. Moreover, it is not required that every node be accessible from a single root node via a feature path; instead, it is required that every node be accessible from one of the base-labelled nodes. Semantic frames defined in this way can be seen as finite first-order structures which conform to a signature consisting of a set LabelUType of unary relation symbols and 
a set FeatURel of binary relation symbols subject to the constraints that the members of Label denote singletons, the members of Feat denote functional relations, and that the above accessibility condition holds. In the example frame of Figure 1 , symbols inside nodes $\left(l_{0}, l_{1}, \ldots\right)$ indicate base labels, symbols attached to nodes (man, motion, ...) belong to Type, members of Feat are marked by small caps (AGENT, ENDP, ...), and part-of is the only member of Rel occurring in this frame.

But the logical framework of (Kallmeyer and Osswald 2013) does not provide means for explicit quantification. As a consequence, the referential entities of the domain of discourse are implicitly treated as definite, which is reflected by the naming of nodes $l_{0}, l_{1}$, etc.

Such relational structures can also easily be turned into Kripke structures. Thus, semantic frames, or feature structures, provide a natural application domain for modal languages and, in particular, for hybrid extensions because of the need to cope with node labels and feature path re-entrancies (Blackburn 1993).

As Blackburn (1993) points out, attribute-value structures can be described using the logical language of Hybrid Logic (HL, cf. Areces and ten Cate 2007), an extension of the language of modal logic, wellsuited to the description of graph structures like the one of Figure 1. HL introduces nominals, i.e., node names, that allow the logical formulas to refer to specific nodes of the graph. The nominal $l_{0}$ for instance refers to the motion node in Figure 1. It is then possible, for example, to specify that the AGENT and the MOVER edges from the node $l_{0}$ should meet on the same node in Figure 1. This additional expressiveness of HL over modal logic allows one to express node sharing in attribute value structures (Blackburn 1993). HL is an established logical formalism which has been extensively studied, in particular with respect to the addition of variables for nodes, and the associated binders, that can appear in the logical formulas. Its relation to attribute-value structures and its expressiveness make it a natural candidate to relate quantified expressions and frame semantics.

With respect to Kallmeyer and Osswald (2013), the approach we propose here does not consider frames as "genuine semantic representations". The one-to-one equivalence between the logical formulas of the LAVD language of Kallmeyer and Osswald (2013) and the frames 
as graph (or relational) structures relies on the existence of minimal models for such formulas. While HL with nominals but without variables nor binders is very close to the LAVD language, it is not obvious what the notion of minimal model of the latter becomes when using quantification. Thus, we have a more traditional view where the sense of an expression is a hybrid logical formula and its reference is computed against models. The latter are the frames we wish to consider. But, contrary to what happens with minimal models, they are then not fully specified by the logical formulas which serve as frame descriptions.

This move from encodings to models is closely related to the one from feature value matrices, as directly encoding a graph, to descriptions of admissible structures when negation or disjunction were introduced (Blackburn 1993, see the end of Section 1).

\section{Related work}

Hybrid logic with nominals but without quantification over states was already used to describe semantic dependency graphs by Baldridge and Kruijff (2002). Natural language quantification is there encoded using RESTR and BODY relations. However, it remains unclear how to compute relations between such representations (e.g., how to check that John kisses Mary holds in case every man kisses Mary holds). An additional step of interpretation of the graphs seems to be required.

A similar approach is proposed by Kallmeyer and Richter (2014) for quantification in frame semantics. In this approach, "quantifier frames" also introduce RESTR and BODY attributes that point to nodes (typically representing an entity and an event, respectively). But they do not directly encode the truth conditions that would be associated with a model-theoretic interpretation. Bridging the gap between the quantifier frame and the model-theoretic interpretation requires the additional extraction of a predicate-logical formula; this, in turn, can be model-theoretically interpreted in order to compute the truth value of the expression.

Baldridge and Kruijff (2002) and Kallmeyer and Richter (2014) consider frames to be expressions that need to be further interpreted, possibly as formulas of predicate logic that, in turn, can be given a model-theoretic interpretation in a usual way. 
In the approach we presently propose, and contrary to these previous approaches, there is no quantifier frame as such. The frames themselves are the models. The quantifiers are part of the formulas describing or constraining the frames (as models of the logical formulas) that can make an expression true. The HL formulas are the expressions to be interpreted as frames. But for the latter, no additional interpretation is required. The logical operators and the frames (as models) are kept separate, following the approach suggested by Muskens (2013).

\section{HYBRID LOGIG AND SEMANTIC FRAMES}

2.1 Hybrid logic

We use the notations of Areces and ten Cate (2007).

Definition 1 (Formulas). Let Rel = Func $\cup$ PropRel be a set of functional and non-functional relational symbols, Prop a set of propositional variables, Nom a set of nominals (node names), and Svar a set of state variables. Let Stat $=$ Nom $\cup$ Svar.

The language of formulas Forms is defined as:

$$
\text { Forms ::= }=\top|p| s|\neg \phi| \phi_{1} \wedge \phi_{2}|\langle R\rangle \phi| \exists \phi\left|@_{s} \phi\right| \downarrow x . \phi \mid \exists x . \phi
$$

where $p \in$ Prop, $s \in$ Stat, $R \in$ Rel, $x \in$ Svar, and $\phi, \phi_{1}, \phi_{2} \in$ Forms.

Moreover, we define:

• $\forall \phi \equiv \neg \exists \neg \phi$

- $[R] \phi \equiv \neg\langle R\rangle \neg \phi$

- $\phi \Rightarrow \psi \equiv \neg \phi \vee \psi$

We call $\forall$ and $[R]$ universal operators, and $\exists$ and $\langle R\rangle$ existential operators. The elements of Func will be written in small caps.

The $\langle R\rangle$ and $[R]$ operators are the usual modal operators corresponding to some accessibility relation $R$. The semantics of the new operators are given in the definitions to come, but the intuition behind them is as follows. The $\exists \phi$ formula states that somewhere in the relational structure there is a node where $\phi$ holds. $\forall \phi$ holds only if $\phi$ holds at each node of the structure. The binder $\downarrow$ in $\downarrow x$. $\phi$ gives the name $x$ to the current node, so that $x$ can be referred to arbitrarily deep in $\phi$. The quantifier $\exists x$. $\phi$ does not change the evaluation node, 
but states that some other node in the structure exists, is given the name $x$, and is such that $\phi$ (that possibly refers to $x$ ) is true at the current evaluation node. Finally, $@_{s} \phi$ states that $\phi$ holds at the node named $s$. With this operator, it can be checked from any place in the relational structure that the property $\phi$ holds at this specific node.

Definition 2 (Model). A model $\mathscr{M}$ is a triple $\left\langle M,\left(R^{\mathscr{M}}\right)_{R \in \operatorname{Rel}}, V\right\rangle$ such that $M$ is a non-empty set, each $R^{\mathscr{M}}$ is a binary relation on $M$, and the valuation $V:$ Prop $\cup$ Nom $\longrightarrow \wp(M)$ is such that if $i \in$ Nom then $V(i)$ is a singleton. An assignment $g$ is a mapping $g:$ Svar $\longrightarrow M$. For an assignment $g, g_{m}^{x}$ is an assignment that differs from $g$ at most on $x$ and $g_{m}^{x}(x)=m$. For $s \in$ Stat, we also define $[s]^{\mathcal{M}, g}$ to be the only $m$ such that $V(s)=\{m\}$ if $s \in$ Nom and $[s]^{\mathscr{M}, g}=g(s)$ if $s \in$ Svar.

Definition 3 (Satisfaction relation). Let $\mathscr{M}$ be a model, $w \in M$, and $g$ an assignment for $\mathscr{M}$. The satisfaction relation is defined as follows:

$$
\begin{array}{ll}
\mathscr{M}, g, w \vDash \top & \text { iff } w=[s]^{\mathscr{M}, g} \text { for } s \in \text { Stat } \\
\mathscr{M}, g, w \vDash s & \text { iff } \mathscr{M}, g, w \vDash \phi \\
\mathscr{M}, g, w \vDash \neg \phi & \text { iff } \mathscr{M}, g, w \vDash \phi_{1} \text { and } \mathscr{M}, g, w \vDash \phi_{2} \\
\mathscr{M}, g, w \vDash \phi_{1} \wedge \phi_{2} & \text { iff there is a } w^{\prime} \in M \text { such that } \\
\mathscr{M}, g, w \vDash\langle R\rangle \phi & R^{\mathscr{M}}\left(w, w^{\prime}\right) \text { and } \mathscr{M}, g, w^{\prime} \vDash \phi \\
\mathscr{M}, g, w \vDash p & \text { iff } w \in V(p) \text { for } p \in \text { Prop } \\
\mathscr{M}, g, w \vDash @_{s} \phi & \text { iff } \mathscr{M}, g,[s]^{\mathscr{M}, g} \phi \phi \text { for } s \in \text { Stat } \\
\mathscr{M}, g, w \vDash \downarrow x . \phi & \text { iff } \mathscr{M}, g_{w}^{x}, w \vDash \phi \\
\mathscr{M}, g, w \vDash \exists x . \phi & \text { iff there is a } w^{\prime} \in M \text { such that } \mathscr{M}, g_{w^{\prime}}^{x}, w \vDash \phi \\
\mathscr{M}, g, w \vDash \exists \phi & \text { iff there is a } w^{\prime} \in M \text { such that } \mathscr{M}, g, w^{\prime} \vDash \phi
\end{array}
$$

We can then check that $\mathscr{M}, g, w \vDash \forall \phi$ iff $\forall w^{\prime} \mathscr{M}, g, w^{\prime} \vDash \phi . \forall$ is the universal modality. $\forall \phi$ states that the property $\phi$ should hold at each node of the model.

Definition 4 (Satisfaction and validity). A formula $\phi$ is:

- satisfiable if there is a model $\mathscr{M}$, and an assignment $g$ on $\mathscr{M}$, and a state $w \in M$ such that $\mathscr{M}, g, w \vDash \phi$

- globally true in a model $\mathscr{M}$ under an assignment $g$ if it is satisfiable at all states of the model, i.e., $\mathscr{M}, g, w \vDash \phi$ for all $w \in M$. We write $\mathscr{M}, g \vDash \phi$

- valid if for all models $\mathscr{M}$ and assignments $g, \mathscr{M}, g \vDash \phi$. 
We can reformulate the frame of Figure 1 (Section 1) within this framework. The Prop vocabulary we use in the HL formulas corresponds to the unary relation symbols of Type used by Kallmeyer and Osswald (2013) to represent frames (see Section 1.1). The Nom vocabulary corresponds to the unary relation symbols of Label, and the Rel vocabulary subsumes the Feat binary relations of Kallmeyer and Osswald (2013). Note that the functionality of the members of Feat must be enforced separately by axioms. The semantic frame of Figure 1 is then a model that satisfies the formula (1) at the element named by $l_{0}$. This formula also highlights the crucial role of nominals in this setting. Several other formulas would be possible, but using an @ operator, here $@_{v}$, is needed to specify that the AT-REGION and the part-of edges meet on the same node (the $@_{l_{2}}$ is not completely necessary to describe the structure of Figure 1 but naturally arises in a compositional computing of this representation).

$$
\begin{aligned}
& l_{0} \wedge \text { motion } \wedge\langle\text { AGENT }\rangle\left(l_{1} \wedge \text { man }\right) \wedge\langle\text { MOVER }\rangle l_{1} \wedge \\
&\langle\text { GOAL }\rangle\left(l_{2} \wedge \text { house }\right) \wedge\langle\text { MANNER }\rangle \text { walking } \wedge \\
&(\exists v w .\langle\text { PATH }\rangle(\text { path }\wedge\langle\text { ENDP }\rangle) \wedge \\
&\left.@_{l_{2}}(\langle\text { AT }- \text { REGION }\rangle w) \wedge @_{v}(\langle\text { part-of }\rangle w)\right)
\end{aligned}
$$

Expressive power

According to the satisfaction relation definition, $\downarrow$ and $\exists$ bind node variables without changing the current evaluation node. In addition to $\exists$, Blackburn and Seligman (1995) introduce another quantifier $\Sigma$ for which the satisfaction relation also changes the evaluation node: ${ }^{1}$

$$
\mathscr{M}, g, w \vDash \Sigma x . \phi \text { iff } \exists w^{\prime} \mathscr{M}, g_{w^{\prime}}^{x}, w^{\prime} \vDash \phi
$$

This defines two independant families of operators: $\downarrow$ and $\exists$, and $\exists$ and $\Sigma .^{2}$ However, using any two operators of both families (for instance $\downarrow$ and $\exists$, the "weakest" ones) is expressively equivalent to using the most expressive fragment of the hybrid languages (the full hybrid language).

\footnotetext{
${ }^{1}$ Blackburn and Seligman (1995) call $\exists$ the somewhere operator, and write it $\diamond$, and $\forall$ is the universal modality, written $\square$.

${ }^{2}$ Note that $\downarrow$ can be defined in terms of $\exists$ by $\downarrow x . \phi \equiv \exists x . x \wedge \phi$ and that $\exists$ can be defined in terms of $\Sigma$ by $\exists \phi \equiv \Sigma z$. $\phi$ with $z$ not occurring in $\phi$.
} 
It is usual to refer to the hybrid languages $\mathscr{H}\left(\theta_{1}, \ldots, \theta_{n}\right)$ as the extension of the modal language with nominals and the operators $\theta_{1}, \ldots, \theta_{n} \in\{\downarrow @, \exists, \exists\}$. It is worth noting that even using the simplest binder $\downarrow$ already causes the satisfiability problem for $\mathscr{H}(\downarrow)$ to be undecidable (Areces et al. 1999) where the satisfiability problem corresponds to answering the question whether given a formula $\phi$, there is a model $\mathscr{M}$, an assignment $g$ and a node $w$ such that $\mathscr{M}, g, w \vDash \phi$.

Nevertheless, there are syntactic restrictions on formulas that make the satisfiability problem decidable. In particular, formulas of the full hybrid language that do not contain the pattern "universal operator scoping over a $\downarrow$ operator scoping over a universal operator" have a decidable satisfiability problem (ten Cate and Franceschet 2005). Such formulas are used by Kallmeyer et al. (2015).

But the formulas we use in the present paper do show this pattern. On the other hand, they do not use the pattern "existential operator scoping over a $\downarrow$ operator scoping over an existential operator". For such formulas, the validity problem is shown to be decidable (ten Cate and Franceschet 2005). Although the validity problem for first-order logic is undecidable, this result by itself does not really improve on first-order logic representations. A more promising approach would be to consider semantic restrictions of the underlying class of models. For instance, (Schneider 2007) describes some classes where decidability results hold. As we do not take advantage so far of the Frame Semantics hypothesis that considers attributes to be functional, the class of models with such a semantic restriction is a natural candidate for studying the satisfiability problem. In any case, for every hybrid language, testing a given formula against a given finite model is decidable (Franceschet and de Rijke 2006).

Since the models we are considering are semantic frames instead of arbitrary first-order models, we first present some models in which we consider the sentences (2a), (3a), and (4a). When the model is the frame of Figure 2, we expect (2a) to be true. There indeed is a kissing event with AGENT and THEME attributes linking to persons named (represented by the NAME attribute) John and Mary respectively. Accordingly, we wish to represent the semantics of (2a) by the hybrid logic formula (2b). 
Quantification in frame semantics

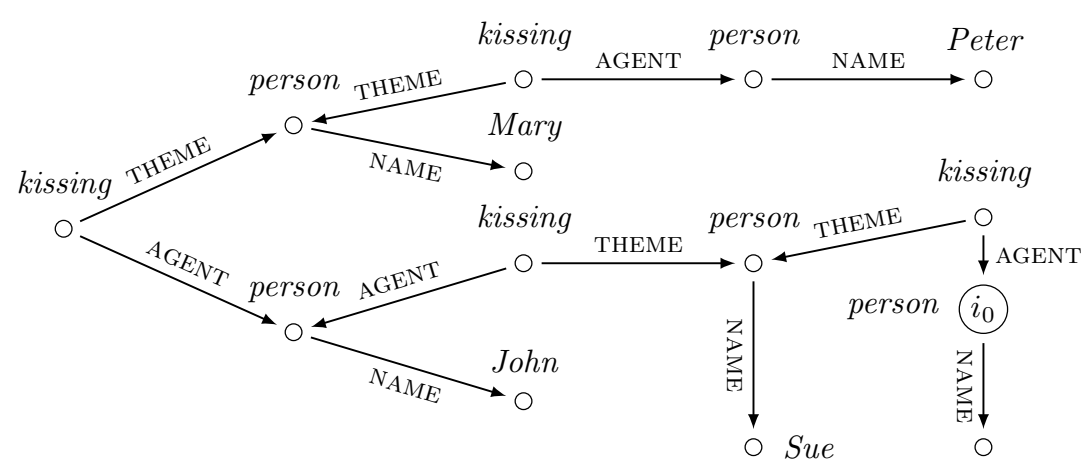

Figure 2:

Quantification

Paul

On the other hand, (3a) is expected to be false as there is a person named Paul who is AGENT of a single kissing event whose THEME is a person named Sue. The frame of Figure 2 indeed falsifies the formula (3b) because we can find a node (namely, $i_{0}$ ) at which man holds, ${ }^{3}$ but there is no kissing node from which we can both reach $i_{0}$ through an AGENT relation and, through a THEME relation, a node at which person $\wedge\langle$ NAME $\rangle$ Mary also holds.

With the object wide scope reading, we also expect (4a) to be false in the frame of Figure 2 because while the person named Paul and Peter both are AGENT of kissing events, these events do not have the same THEME. However, with the subject wide scope reading, (4a) is expected to be true in this frame.

(2) a. John kisses Mary

b. $\exists($ kissing $\wedge\langle$ AGENT $\rangle($ person $\wedge\langle$ NAME $\rangle$ John $) \wedge$

$\langle$ THEME $\rangle($ person $\wedge\langle$ NAME $\rangle$ Mary $))$

(3) a. Every man kisses Mary

b. $\forall(\downarrow$ i.man $\Rightarrow \exists($ kissing $\wedge\langle$ AGENT $\rangle i \wedge$

$\langle$ THEME $\rangle($ person $\wedge\langle$ NAME $\rangle$ Mary $))$ )

(4) a. Every man kisses some woman

b. $\forall\left(\downarrow\right.$ i.man $\Rightarrow \exists\left(\downarrow i^{\prime}\right.$.woman $\wedge$

$\exists\left(k i s s i n g \wedge\langle\right.$ AGENT $\rangle i \wedge\langle$ THEME $\left.\left.\left.\rangle i^{\prime}\right)\right)\right)$

c. $\exists\left(\downarrow\right.$ i.woman $\wedge \forall\left(\downarrow i^{\prime}\right.$.man $\Rightarrow$

$\exists\left(k i s s i n g \wedge\langle\right.$ AGENT $\rangle i^{\prime} \wedge\langle$ THEME $\left.\left.\left.\rangle i\right)\right)\right)$

\footnotetext{
${ }^{3}$ Actually, in Figure 2, only person holds at $i_{0}$. We can have man hold as well with the additional postulate that (person $\wedge\langle\mathrm{NAME}\rangle$ Paul $) \Rightarrow$ man, and similarly of each node with a NAME attribute.
} 
Figure 3:

Quantification and node sharing

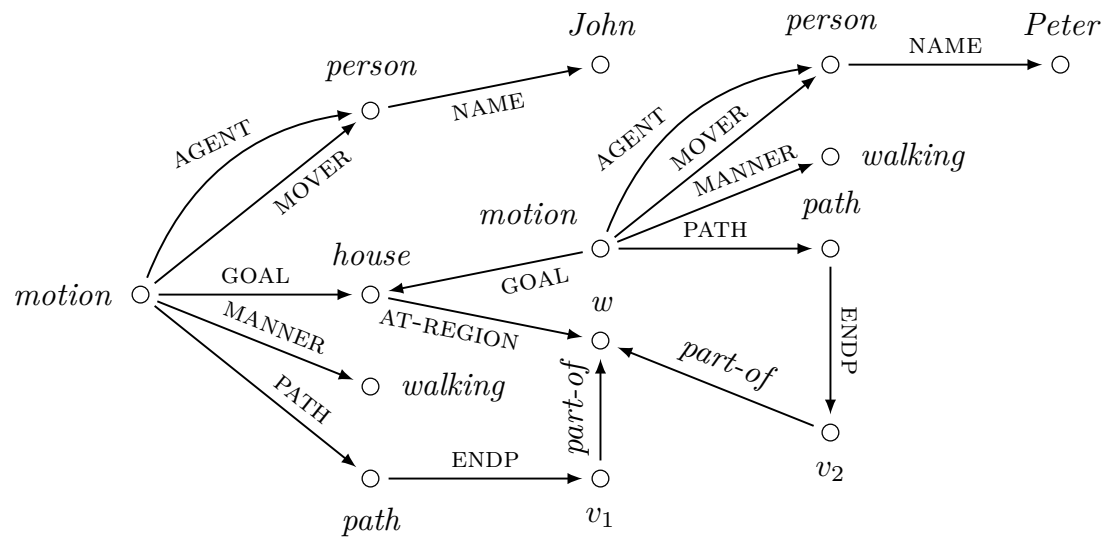

(5a) shows how state storing with the $\downarrow$ operator correctly interacts with the @ operator in order to describe node sharing. This sentence is expected to be true (both readings) in the model given by the frame of Figure 3.The frame semantics analysis of bounded motions verbs in (Kallmeyer and Osswald 2013) requires the motion to have a GOAL attribute. It is moreover required that the node reached is the same as the one of the entity provided by the $P P$. We express this requirement in the HL formulas (5b) and (5c):

1 . by binding to the variable $i^{\prime}$ a house node,

2. by binding to the variable $g$ a node that is accessible from the motion node via the $\langle\mathrm{GOAL}\rangle$ relation,

3. and by stating that $i^{\prime}$ and $g$ should be the same node, i.e., $g \wedge i^{\prime}$ should hold.

(5) a. Every man walked to some house

b. $\quad \forall\left(\downarrow\right.$ i.man $\Rightarrow\left(\exists\left(\downarrow i^{\prime}\right.\right.$.house $\wedge$

$(\exists a g . \exists($ motion $\wedge\langle$ AGENT $\rangle a \wedge\langle$ MOVER $\rangle a \wedge\langle$ GOAL $\rangle \wedge \wedge$

$\langle$ PATH $\rangle$ path $\wedge\langle$ MANNER $\rangle$ walking $\wedge @_{a} i \wedge$

$(\exists r v$ w.event $\wedge\langle\mathrm{PATH}\rangle($ path $\wedge\langle\mathrm{ENDP}\rangle) \wedge$

$@_{r}(\langle$ AT-REGION $\rangle w) \wedge @_{v}(\langle$ part-of $\left.\left.\left.\left.\left.\left.\rangle w) \wedge @_{r}\left(g \wedge i^{\prime}\right)\right)\right)\right)\right)\right)\right)$

c. $\exists\left(\downarrow i^{\prime}\right.$.house $\wedge(\forall(\downarrow$ i.man $\Rightarrow$

$(\exists a g . \exists($ motion $\wedge\langle$ AGENT $\rangle a \wedge\langle$ MOVER $\rangle a \wedge\langle$ GOAL $\rangle \wedge \wedge$

$\langle$ PATH $\rangle$ path $\wedge\langle$ MANNER $\rangle$ walking $\wedge @_{a} i \wedge$

$(\exists r v$ w.event $\wedge\langle\mathrm{PATH}\rangle($ path $\wedge\langle\mathrm{ENDP}\rangle) \wedge$

$@_{r}(\langle$ AT-REGION $\rangle w) \wedge @_{\nu}(\langle$ part-of $\left.\left.\left.\left.\left.\left.\rangle w) \wedge @_{r}\left(g \wedge i^{\prime}\right)\right)\right)\right)\right)\right)\right)$ 
Our goal is to compositonally associate each expression in natural language to an HL formula. This logical formula is to be checked against the possible models, and the sentence is true w.r.t. a model $\mathscr{M}$ in case this model satisfies the logical formula. More precisely, given a sentence $s$ and its semantic representation $\llbracket s \rrbracket$, we say that $s$ is true iff for all assignments $g, \mathscr{M}, g \vDash \llbracket s \rrbracket$ (i.e., $\llbracket s \rrbracket$ is globally true in $\mathscr{M}$ under any assignment).

Note that we use several modal operators. Each of them describes the accessibility relations corresponding to one of the attributes we find in frames (AGENT, GOAL, etc.). They should not be confused with other possible modal operators that are used for natural language semantics (e.g., knowledge and belief, intensionality, etc.). Clarifying the interaction between these different kinds of modal operators, for instance following Blackburn and Rijke (1997), is an important issue. But this goes beyond the scope of this paper and is left for future work.

3

\section{SYNTAX-SEMANTICS INTERFACE WITH}

ABSTRACT GATEGORIAL GRAMMARS

In order to exemplify our approach to quantification in frame semantics, we rely on the framework of Abstract Categorial Grammars (ACG) (de Groote 2001). ACGs derive from type-theoretic grammars in the tradition of Lambek (1958), Curry (1961), and Montague (1974). Rather than being a grammatical formalism on their own, they provide a framework in which several grammatical formalisms may be encoded (de Groote and Pogodalla 2004). Since our focus is on the semantic modelling of quantification in frame semantics and its compositional account, we provide a Montague grammar based syntactic modelling that is sufficient for our purpose. Integration of the modelling of scope ambiguity in a TAG encoding (de Groote 2002) for instance would require an embedding into an underspecified representation language (Bos 1995; Pogodalla 2004; Kallmeyer and Romero 2008) that plays no role in the final interpretation of the logical formula to be interpreted.

\section{Abstract Categorial Grammars}

The definition of an ACG is based on a small set of mathematical primitives from type theory, $\lambda$-calculus, and linear logic. These primitives 
combine via simple composition rules, offering ACGs good flexibility. In particular, ACGs generate languages of linear $\lambda$-terms, which generalize both string and tree languages. Crucially, ACG provides the user with direct control over the parse structures of the grammar, the abstract language. Such structures are later interpreted by a morphism, the lexicon, to get the concrete object language. A vocabulary is the higher-order signature that defines the atomic elements (atomic types and typed constants).

For sake of self-containedness, we review here the basic definitions of ACGs.

Definition 5 (Types). Let $A$ be a set of atomic types. The set $\mathscr{T}(A)$ of implicative types built upon $A$ is defined with the following grammar:

$$
\mathscr{T}(A)::=A|\mathscr{T}(A) \rightarrow \mathscr{T}(A)| \mathscr{T}(A) \rightarrow \mathscr{T}(A)
$$

The set of linear implicative types built upon $A$ is defined with the following grammar:

$$
\mathscr{T}^{0}(A)::=A \mid \mathscr{T}^{0}(A) \rightarrow \mathscr{T}^{0}(A)
$$

Definition 6 (Higher-order signatures). A higher-order signature $\Sigma$ is a triple $\Sigma=\langle A, C, \tau\rangle$ where:

- $A$ is a finite set of atomic types;

- $C$ is a finite set of constants;

- $\tau: C \rightarrow \mathscr{T}(A)$ is a function assigning types to constants.

A higher-order signature $\Sigma=\langle A, C, \tau\rangle$ is linear if the codomain of $\tau$ is $\mathscr{T}^{0}(A)$.

Definition 7 ( $\lambda$-Terms). Let $X$ be an infinite countable set of $\lambda$ variables. The set $\Lambda(\Sigma)$ of $\lambda$-terms built upon a higher-order signature $\Sigma=\langle A, C, \tau\rangle$ is inductively defined as follows:

- if $c \in C$ then $c \in \Lambda(\Sigma)$;

- if $x \in X$ then $x \in \Lambda(\Sigma)$;

- if $x \in X$ and $t \in \Lambda(\Sigma)$ and $x$ occurs free in $t$ exactly once, then $\lambda^{\mathrm{o} x} . t \in \Lambda(\Sigma)$

- if $x \in X$ and $t \in \Lambda(\Sigma)$, then $\lambda x . t \in \Lambda(\Sigma)$;

- if $t, u \in \Lambda(\Sigma)$ then $(t u) \in \Lambda(\Sigma)$. 
Note there is a linear $\lambda$-abstraction (denoted by $\lambda^{0}$ ) and a (usual) intuitionistic $\lambda$-abstraction (denoted by $\lambda$ ). There also are the usual notions of $\alpha, \beta$, and $\eta$ conversions (Barendregt 1984).

Definition 8 (Typing judgment). Given a higher-order signature $\Sigma$, the typing rules are given with an inference system whose judgments are of the form: $\Gamma ; \Delta \vdash_{\Sigma} t: \alpha$ where:

- $\Gamma$ is a finite set of non-linear variable typing declarations;

- $\Delta$ is a finite set of linear variable typing declarations.

Both $\Gamma$ and $\Delta$ may be empty. If both of them are empty, we usually write $t: \alpha(t$ is of type $\alpha)$ instead of $\vdash_{\Sigma} t: \alpha$. Moreover, we drop the $\Sigma$ subscript when the context permits. Table 1 gives the typing rules.

$$
\begin{array}{lc}
\frac{\Gamma ; \vdash_{\Sigma} c: \tau(c)}{\text { (const.) }} \\
\frac{\Gamma ; x: \alpha ; \vdash_{\Sigma} x: \alpha}{\Gamma ; x \vdash_{\Sigma} x: \alpha} \text { (lin. var.) } \\
\frac{\Gamma ; \Delta, x: \alpha \vdash_{\Sigma} t: \beta}{\Gamma ; \Delta \vdash_{\Sigma} \lambda^{\mathbf{0} x . t: \alpha \rightarrow \beta}} \text { (1. abs.) } \frac{\Gamma ; \Delta_{1} \vdash_{\Sigma} t: \alpha \rightarrow \beta \quad \Gamma ; \Delta_{2} \vdash_{\Sigma} u: \alpha}{\Gamma ; \Delta_{1}, \Delta_{2} \vdash_{\Sigma}(t u): \beta} \text { (l. app.) } \\
\frac{\Gamma, x: \alpha ; \Delta \vdash_{\Sigma} t: \beta}{\Gamma ; \Delta \vdash_{\Sigma} \lambda x . t: \alpha \rightarrow \beta} \text { (abs.) } \frac{\Gamma ; \Delta \vdash_{\Sigma} t: \alpha \rightarrow \beta \quad \Gamma ; \vdash_{\Sigma} u: \alpha}{\Gamma ; \Delta \vdash_{\Sigma}(t u): \beta} \text { (app.) }
\end{array}
$$

Remark. In the rule (app.), the linear context needs to be empty. Otherwise, a linear variable occurring in $u$ could be duplicated or removed if the non-linear abstracted variable in $t$ for which it substitutes in a $\beta$-reduction is duplicated or removed.

Definition 9 (Lexicon). Let $\Sigma_{1}=\left\langle A_{1}, C_{1}, \tau_{1}\right\rangle$ and $\Sigma_{2}=\left\langle A_{2}, C_{2}, \tau_{2}\right\rangle$ be two higher-order signatures, $\Sigma_{1}$ being linear. A lexicon $\mathscr{L}=\langle F, G\rangle$ from $\Sigma_{1}$ to $\Sigma_{2}$ is such that:

- $F: A_{1} \rightarrow \mathscr{T}\left(A_{2}\right)$. We also note $F: \mathscr{T}^{0}\left(A_{1}\right) \rightarrow \mathscr{T}\left(A_{2}\right)$ its homomorphic extension; ${ }^{4}$

- $G: C_{1} \rightarrow \Lambda\left(\Sigma_{2}\right)$. We also note $G: \Lambda\left(\Sigma_{1}\right) \rightarrow \Lambda\left(\Sigma_{2}\right)$ its homomorphic extension;

\footnotetext{
${ }^{4}$ Such that $F(\alpha \rightarrow \beta)=F(\alpha) \rightarrow F(\beta)$ and $F(\alpha \rightarrow \beta)=F(\alpha) \rightarrow F(\beta)$
} 
- $F$ and $G$ are such that for all $c \in C_{1}, \quad \vdash_{\Sigma_{2}} G(c): F\left(\tau_{1}(c)\right)$ is provable.

We also use $\mathscr{L}$ instead of $F$ or $G$.

Definition 10 (Abstract Categorial Grammar and vocabulary). An $a b$ stract categorial grammar is a quadruple $\mathscr{G}=\left\langle\Sigma_{1}, \Sigma_{2}, \mathscr{L}, S\right\rangle$ where:

- $\Sigma_{1}=\left\langle A_{1}, C_{1}, \tau_{1}\right\rangle$ and $\Sigma_{2}=\left\langle A_{2}, C_{2}, \tau_{2}\right\rangle$ are two higher-order signatures and $\Sigma_{1}$ is linear. $\Sigma_{1}$ is called the abstract vocabulary and $\Lambda\left(\Sigma_{1}\right)$ is the set of abstract terms; similarly, $\Sigma_{2}$ is called the object vocabulary and $\Lambda\left(\Sigma_{2}\right)$ is the set of object terms.

- $\mathscr{L}: \Sigma_{1} \rightarrow \Sigma_{2}$ is a lexicon.

- $S \in \mathscr{T}\left(A_{1}\right)$ is the distinguished type of the grammar.

Given an ACG $\mathscr{G}_{\text {name }}=\left\langle\Sigma_{1}, \Sigma_{2}, \mathscr{L}_{\text {name }}, S\right\rangle$, we use the following notational variants for the interpretation of the type $\alpha$ (resp. the term t): $\mathscr{L}_{\text {name }}(\alpha)=\beta, \mathscr{G}_{\text {name }}(\alpha)=\beta, \alpha:=_{\text {name }} \beta$, and $\llbracket \alpha \rrbracket_{\text {name }}=\beta$ (resp. $\mathscr{L}_{\text {name }}(t)=u, \mathscr{G}_{\text {name }}(t)=u, t:=_{\text {name }} u$, and $\left.\llbracket t \rrbracket_{\text {name }}=u\right)$. The subscript may be omitted if clear from the context.

Definition 11 (Abstract and object languages). Given an ACG $\mathscr{G}$, the abstract language is defined by

$$
\mathscr{A}(\mathscr{G})=\left\{t \in \Lambda\left(\Sigma_{1}\right) \mid \vdash_{\Sigma_{1}} t: S \text { is derivable }\right\}
$$

The object language is defined by

$$
\mathscr{O}(\mathscr{G})=\left\{u \in \Lambda\left(\Sigma_{2}\right) \mid \exists t \in \mathscr{A}(\mathscr{G}) \text { s.t. } u=\mathscr{L}(t)\right\}
$$

\section{The syntax-semantics interface as ACG composition}

The lexicon defines the way structures are interpreted. It plays a crucial role in the way ACG models the syntax-semantics interface. The basic idea is to have a given (abstract) structure interpreted either as a surface form (e.g., a string) or as a meaning form (e.g., a logical formula). This boils down to having two interpretations that share the same abstract vocabulary, hence mapping a single structure into two different ones. This composition is illustrated by $\mathscr{G}_{\text {form }}$ and $\mathscr{G}_{\text {meaning }}$ sharing the $\Sigma_{\text {abstract }}$ vocabulary in Figure 4. 


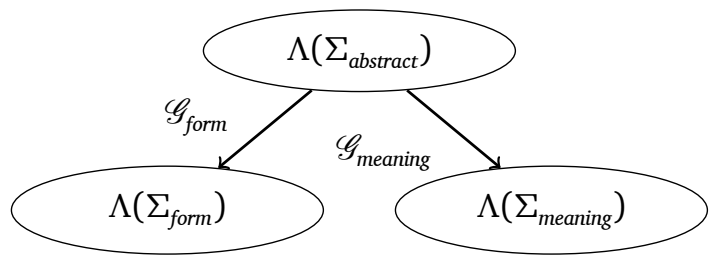

Figure 4:

ACG composition for the syntax-semantics interface

\section{TYPE-THEORETIC SEMANTICS WITH FRAMES}

We now provide the type-theoretic syntax-semantics interface allowing for a compositional building of the meanings. We use the architecture described in Figure 4. As we are concerned in this article with semantic modelling and quantification rather than with parsing, we use higher-order types for quantified noun-phrases.

All the following examples can be run and tested with the ACG toolkit ${ }^{5}$ and the companion example files. ${ }^{6}$

\section{The ACG of surface forms}

At the abstract level, we use the signature defined with the type assignment of Table 2. It makes use of the usual syntactic types: $N P, S$, $N$, and $P P$. Note that following the usual type-logical approach, determiners have a higher-order type.

$$
\begin{aligned}
\text { John, Mary } & : N P & \text { kisses } & : N P \rightarrow N P \rightarrow S \\
\text { man, woman, house } & : N & \text { every, some } & : N \rightarrow(N P \rightarrow S) \rightarrow S \\
\text { to, into } & : N P \rightarrow P P & \text { walked } & : P P \rightarrow N P \rightarrow S
\end{aligned}
$$

Table 2:

$\Sigma_{\text {abstract }}$ type assignement

The object vocabulary of surface forms uses the standard modelling of strings as $\lambda$-terms. It is built on $\Sigma_{\text {form }}$ that contains a single atomic type $o$, and the type $\sigma$ (for strings) is defined by $\sigma \triangleq o \rightarrow o$. The concatenation is then defined as functional composition by $\cdot+\cdot=$ $\lambda^{\mathrm{o}} f g . \lambda^{\mathrm{o}} z . f(g z): \sigma \rightarrow \sigma \rightarrow \sigma$. It is associative, and it admits the identity function $\epsilon \triangleq \lambda^{\mathrm{o}} x . x: \sigma$ as a neutral element. $\Sigma_{\text {form }}$ also contains the constants John, Mary, kisses, every, man ... of type $\sigma$.

The ACG $\mathscr{G}_{\text {form }}$ is then defined using the interpretations given in Table 3. The terms defined in Equations (6) correspond to the syntactic

\footnotetext{
${ }^{5}$ ACGtk can be downloaded and installed from http://calligramme. loria.fr/acg/\#Software.

${ }^{6}$ These files are available at https://hal.inria.fr/hal-01417853/ file/quantification-and-frames.zip.
} 
derivations of the sentence for which we want to provide a semantic representation. Their surface forms are given by Equations (7)-(12).

$$
\begin{aligned}
& u_{2 \mathrm{~b}}=\text { kisses Mary John } \\
& u_{3 \mathrm{~b}}=(\text { every man })\left(\lambda^{\mathrm{o}} x \text {.kisses Mary } x\right) \\
& \left.u_{4 \mathrm{~b}}=(\text { every man })\left(\lambda^{\mathrm{o}} x \text {. (some woman }\right)\left(\lambda^{\mathrm{o}} y \text {.kisses } y x\right)\right) \\
& \left.u_{4 \mathrm{c}}=(\text { some woman })\left(\lambda^{\mathrm{o}} y \text {. (every man }\right)\left(\lambda^{\mathrm{o}} x \text {.kisses } y x\right)\right) \\
& \left.\left.u_{5 \mathrm{~b}}=(\text { every man })\left(\lambda^{\mathrm{o}} x \text {. (some house }\right)\left(\lambda^{\mathrm{o}} y \text {.walked (to } y\right) x\right)\right) \\
& \left.u_{5 \mathrm{c}}=(\text { some house })\left(\lambda^{\mathrm{o}} y \text {. (every man) }\left(\lambda^{\mathrm{o}} x \text {.walked (to } y\right) x\right)\right)
\end{aligned}
$$

$$
\begin{aligned}
& u_{2 \mathrm{~b}}:={ }_{\text {form }} \text { John }+ \text { kisses }+ \text { Mary } \\
& u_{3 \mathrm{~b}}:=_{\text {form }} \text { every }+ \text { man }+ \text { kisses }+ \text { Mary } \\
& u_{4 \mathrm{~b}}:=_{\text {form }} \text { every }+ \text { man }+ \text { kisses }+ \text { some }+ \text { woman } \\
& u_{4 \mathrm{c}}:={ }_{\text {form }} \text { every }+ \text { man }+ \text { kisses }+ \text { some }+ \text { woman } \\
& u_{5 \mathrm{~b}}:={ }_{\text {form }} \text { every }+ \text { man }+ \text { walked }+ \text { to }+ \text { some }+ \text { house } \\
& u_{5 \mathrm{c}}:={ }_{\text {form }} \text { every }+ \text { man }+ \text { walked }+ \text { to }+ \text { some }+ \text { house }
\end{aligned}
$$

Table 3:

$\mathscr{G}_{\text {form }}$ interpretation of the abstract atomic types and

$$
\begin{aligned}
& \text { John }:={ }_{\text {form }} \text { John } \\
& \text { man }:=_{\text {form }} \text { man } \\
& \text { house: }=_{\text {form }} \text { house } \\
& \text { to }:==_{\text {form }} \lambda^{\mathbf{o}} \text { n.to }+n \quad \text { into }:==_{\text {form }} \lambda^{\mathbf{o}} \text { n.into }+n \\
& \text { every }:=_{\text {form }} \lambda^{\mathbf{o}} n \text { P.P }(\text { every }+n) \\
& \text { kisses: }=_{\text {form }} \lambda^{\mathbf{0}} \text { o s.s }+ \text { kissed }+o
\end{aligned}
$$

$$
\begin{aligned}
& \text { Mary }:==_{\text {form }} \text { Mary } \\
& \text { woman }:=_{\text {form }} \text { woman } \\
& \text { into }:=_{\text {form }} \lambda^{\mathbf{o}} \text { n.into }+n \\
& \text { some }:={ }_{\text {form }} \lambda^{\mathbf{0}} n \text { P.P }(\text { some }+n) \\
& \text { walked }:=_{\text {form }} \lambda^{\mathbf{o}} p \text { s.s }+ \text { walked }+p
\end{aligned}
$$

4.2

The ACG of meaning representations

In accordance with the ACG architecture of Figure 4, the syntaxsemantics interface relies on sharing the abstract language of the two ACGs responsible for the surface interpretation on the one hand and for the semantic interpretation on the other hand. The abstract vocabulary we use is $\Sigma_{\text {abstract }}$, defined in the previous section.

Our goal is to associate every sentence with a hybrid-logical formula. It's important to note that we are not concerned with higherorder hybrid logic in this work; not even first-order hybrid logic. The binders and quantifiers we use only bind node variables, and not entities nor higher-order predicates. This contrasts with quantified hybrid 
logic (QHL) (Blackburn and Marx 2002). We do not directly adopt the Hybrid Type Theory (HTT) proposed by Areces et al. (2011, 2014). Contrary to what could be expected from (Gallin 1975) type theory of higher-order modal logic, Areces et al. (2014) do not use a specific type $s$ to denote nodes (or worlds) and nominals are typed $t$ as propositions.

We do introduce a specific type $s$ for nominals, so that the set of atomic types of $\Sigma_{\text {meaning }}$ is $\{s, t\}$. We also introduce a coercion operator \# $: s \rightarrow t$ in order to use nominals as propositions in formulas. This ensures we only build formulas of Forms. Table 4 shows the semantic constants we use, including logical operators and quantifiers.

event, kissing, motion, person, John, Mary, ... : :t

$\langle$ AGENT $\rangle,\langle$ THEME $\rangle,\langle$ MOVER $\rangle,\langle$ part-of $\rangle, \ldots \quad: t \rightarrow t$

$$
\begin{aligned}
\# & : s \rightarrow t \\
\wedge, \Rightarrow & : t \rightarrow t \rightarrow t \\
@ & : s \rightarrow t \rightarrow t \\
\exists, \forall & : t \rightarrow t \\
\downarrow, \exists & :(s \rightarrow t) \rightarrow t
\end{aligned}
$$

Table 4:

Constant terms of the semantic language

We can now define $\mathscr{G}_{\text {meaning }}$ using the interpretations of the atomic types of the constants of Table 5. We follow Kallmeyer and Osswald (2013) in the semantics and meaning decomposition of motion verbs.

$$
\begin{aligned}
& S, N P, N:={ }_{\text {meaning }} t \quad P P:=_{\text {meaning }} t \rightarrow t \\
& \text { John }:={ }_{\text {meaning }} \text { John } \\
& \text { Mary }:={ }_{\text {meaning }} \text { Mary } \\
& \text { man }:={ }_{\text {meaning }} \text { man } \\
& \text { woman }:={ }_{\text {meaning }} \text { woman } \\
& \text { house }:={ }_{\text {meaning }} \text { house } \\
& \text { some }:={ }_{\text {meaning }} \lambda^{\mathrm{o}} P Q . \exists(\downarrow i . P \wedge(Q(\# i))) \\
& \text { every }:={ }_{\text {meaning }} \lambda^{\mathbf{o} P} Q . \forall(\downarrow \text { i.P } \Rightarrow(Q(\# i))) \\
& \text { kisses }:==_{\text {meaning }} \lambda^{\mathrm{o}} \text { os. } . \exists\left(\text { kissing } \wedge\langle\text { AGENT }\rangle_{s} \wedge\langle\text { THEME }\rangle_{o}\right) \\
& \text { walked }:={ }_{\text {meaning }} \lambda^{\mathrm{o}} \text { pp s. } \exists a \text { g. } . \exists(\text { motion } \wedge\langle\operatorname{AGENT}\rangle(\# a) \wedge\langle\operatorname{MOVER}\rangle(\# a) \\
& \wedge\langle\mathrm{GOAL}\rangle(\# g) \wedge\langle\mathrm{PATH}\rangle \text { path } \wedge\langle\text { MANNER }\rangle \text { walking } \\
& \left.\wedge @_{a} s \wedge(p p(\# g))\right) \\
& \text { to } \quad:={ }_{\text {meaning }} \lambda^{\mathrm{o}} n g . \exists r v \text { w.event } \wedge\langle\mathrm{PATH}\rangle(\text { path } \wedge\langle\mathrm{ENDP}\rangle v) \wedge \\
& @_{r}\langle\text { AT-REGION }\rangle(\# w) \wedge @_{v}\langle\text { part-of }\rangle(\# w) \wedge @_{r}(g \wedge n) \\
& \text { into }:={ }_{\text {meaning }} \lambda^{\mathrm{o}} n g . \exists r v \text { w.event } \wedge\langle\mathrm{PATH}\rangle(\text { path } \wedge\langle\mathrm{ENDP}\rangle v) \wedge \\
& @_{r}\langle\text { IN-REGION }\rangle(\# w) \wedge @_{v}\langle\text { part-of }\rangle(\# w) \wedge @_{r}(g \wedge n)
\end{aligned}
$$

Table 5:

Semantic interpretation of the constants of $\Sigma_{\text {abstract }}$ 
Remark. Nominal variables are allowed to occur non-linearly in semantic terms. This is required, for instance, in order to specify that a same nominal is reached from two different paths (see for instance the variable $a$ in $\llbracket$ walked $\rrbracket_{\text {meaning }}$ in Table 5).

What the ACG framework does not express, though, are the lexical or meaning postulates that can be added to the logical theory. Such postulates are additional constraints that any model should also satisfy and that do not depend on the actual semantic representation that is being built. They include for instance the representation of the ontology of propositions (types, in the frame semantics terminology) such as: $\operatorname{man} \Rightarrow$ person, or any standard modal-logical axiom such as $\square(p \Rightarrow q) \Rightarrow(\square p \Rightarrow \square q)$.

It follows that the following equalities hold, where $t_{2 \mathrm{~b}}$ is the term in (2b), $t_{3 \mathrm{~b}}$ is the term in (3b), etc., such that every nominal variable is preceded by the \# coercion operator:

$$
\begin{aligned}
\llbracket \text { kisses Mary John } \rrbracket & =t_{2 \mathrm{~b}} \\
\llbracket(\text { every man })\left(\lambda^{\mathrm{o}} x \text {.kisses Mary } x\right) \rrbracket & =t_{3 \mathrm{~b}} \\
\llbracket(\text { every man })\left(\lambda^{\mathrm{o}} x .(\text { some woman })\left(\lambda^{\mathrm{o}} y \text {. kisses } y x\right)\right) \rrbracket & =t_{4 \mathrm{~b}} \\
\left.\llbracket(\text { some woman })\left(\lambda^{\mathrm{o}} y \text {. (every man }\right)\left(\lambda^{\mathrm{o}} x \text {. kisses } y x\right)\right) \rrbracket & =t_{4 \mathrm{c}}
\end{aligned}
$$

Table 5 shows the interaction of the storing operator with path equalities. It compositionally derives from the verb and the preposition semantic interpretations. In the verb semantics, the path equalities specify that the MOVER and the AGENT attributes of the event are the same, and that the information provided by the $p p$ argument should hold for the GOAL $g$. In its semantics, the preposition contributes on the one hand to the main event (as the event proposition is evaluated at the current state) and on the other hand by specifying that the $g$ state (meant to be the target node of the verb that the proposition modifies, here the target of the GOAL attribute) should be identified to the $n$ argument (the noun phrase which is argument of the preposition). This leads to the interpretations (5b) and (5c) of (5a) given in (17) and (18).

$$
\begin{aligned}
& \left.\left.\llbracket(\text { every man })\left(\lambda^{\mathrm{o}} x \text {. (some house }\right)\left(\lambda^{\mathrm{o}} y \cdot \text { walked (to } y\right) x\right)\right) \rrbracket=t_{5 \mathrm{~b}} \\
& \left.\llbracket(\text { some house })\left(\lambda^{\mathrm{o}} y \text {.(every man) }\left(\lambda^{\mathrm{o}} x \text {.walked (to } y\right) x\right)\right) \rrbracket=t_{5 \mathrm{c}}
\end{aligned}
$$


We now have two ingredients at our disposal: the decomposition of the lexical semantics offered by frame semantics, and the power of binding states. We illustrate how to combine them in order to model semantic coercion. Sentence (19) shows how a predicate can take another event predicate as argument. On the other hand, sentence (20) shows that the same predicate can take a noun phrase as argument. In the latter case, it instead conveys the meaning that the entity referred to by the noun phrase should be part of some event. It is even the case that if this event is not salient in the context, it can be inferred from the lexicon, for instance using the qualia structure and the telic quale as defined by the Generative Lexicon (Pustejovsky 1998), or a subclass of the $\mathbf{S}_{2}$ lexical function in the framework of the Explanatory and Combinatorial Lexicology (Mel'čuk et al. 1995; Polguère 2003).

(19) John began to read a book

(20) John began a book

We first model (19). The assumed syntactic constructions are given by the extension of the $\Sigma_{\text {abstract }}$ signature of Table 6 and by its interpretation by $\mathscr{G}_{\text {form }}$ of Table 7.

Semantically, the idea is that events are structured (Moens and Steedman 1988). We in particular consider the structures required by aspectual predicates such as begin as in (Pustejovsky and Bouillon 1995). We structure the events with the notion of transition that has an ANTE attribute and a POST attribute (see Figure 5). When an event has begun, it is set as the value of the POST attribute. This is what the interpretation of begin $_{1}$ in Table 8 states. This interpretation also requires the event argument to be a process (proc) or an accomplishment (acc) (Im and Lee 2015).

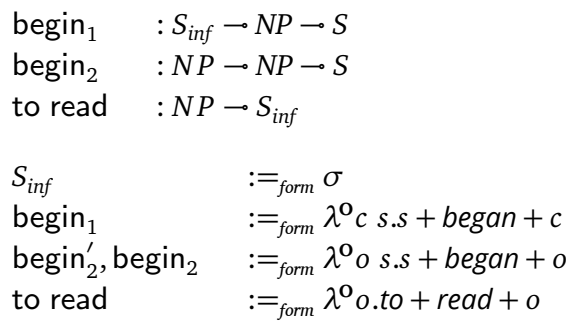

Table 6:

Extension of $\Sigma_{\text {abstract }}$

Table 7:

Interpretation of types and constants of $\Sigma_{\text {abstract }}$ by $\mathscr{G}_{\text {form }}$ 
Figure 5:

Event structure

Table 8:

Interpretation of

types and

constants of

$\Sigma_{\text {abstract }}$ by $\mathscr{G}_{\text {meaning }}$

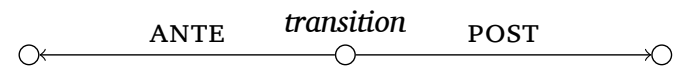

$$
\begin{aligned}
& S_{\text {inf }} \quad:==_{\text {meaning }} t \rightarrow t \\
& \text { begin }_{1} \quad:={ }_{\text {meaning }} \lambda^{\mathbf{0}} c s . \exists(\text { transition } \wedge\langle\mathrm{POST}\rangle((\operatorname{proc} \vee a c c) \wedge(c s))) \\
& \text { begin }_{2}^{\prime} \quad:==_{\text {meaning }} \lambda^{\mathrm{o}} \text { os. } . \exists(\text { transition } \wedge\langle\text { POST }\rangle \\
& \left.\left((\text { proc } \vee \text { acc }) \wedge\langle\mathrm{AGENT}\rangle_{s} \wedge\langle\mathrm{UG}\rangle_{o}\right)\right) \\
& \text { begin }_{2}:=_{\text {meaning }} \lambda^{\mathrm{o}} \text { os. } \exists(\text { transition } \wedge\langle\mathrm{POST}\rangle \\
& (\downarrow s .(p r o c \vee \text { acc }) \wedge\langle\text { AGENT }\rangle s \wedge \\
& \left.\left.\langle\mathrm{UG}\rangle\left(o \wedge\langle\text { proto }\rangle\langle e-q\rangle\left(\downarrow s^{\prime} . @_{s}\langle\text { proto }\rangle\left(\# s^{\prime}\right)\right)\right)\right)\right) \\
& \text { to read }:==_{\text {meaning }} \lambda^{\mathrm{o}} \text { o s.reading } \wedge\langle\text { AGENT }\rangle s \wedge\langle\text { THEME }\rangle_{O}
\end{aligned}
$$$$
\text { (a book) ( } \lambda^{\mathrm{o}} \text { y.begin } \text {. }_{1} \text { (to read } y \text { ) John) }:={ }_{\text {forms }}
$$$$
\text { John }+ \text { began }+ \text { to }+ \text { read }+a+\text { book }
$$$$
\text { (a book) }\left(\lambda^{\mathrm{o}} y \cdot \text { begin }_{1} \text { (to read } y\right) \text { John) }:={ }_{\text {meaning }}
$$$$
\exists(\downarrow \text { i.book } \wedge(\exists(\text { transition }
$$$$
\wedge\langle\mathrm{POST}\rangle((\text { proc } \vee \text { acc }) \wedge \text { reading }
$$$$
\wedge\langle\text { AGENT }\rangle(\text { person } \wedge\langle\text { NAME }\rangle \text { John })
$$$$
\wedge\langle\operatorname{THEME}\rangle(\# i)))) \text { ) }
$$

With the provided ACGs, we can then compute the semantic interpretation of the syntactic derivation associated with (19). Equation (21) shows that the syntactic derivation indeed corresponds to the sentence, and Equation (22) shows its semantic interpretation. In order to be true, the model should have a node $i$ where book holds, and a node where transition holds and from which there is a $\langle\mathrm{POST}\rangle\langle\mathrm{THEME}\rangle$ path to $i$.

It is actually this path that we require to exist in the semantic recipe for begin when used with a direct object. This requirement appears in the interpretation of begin ${ }_{2}^{\prime}$ as given by Table 8 by specifying that the event given as the value of the POST attribute itself has an UNDERGOER (UG) that should target the direct object. This interpretation also accounts for the following constraints (Pustejovsky and Bouillon 1995): the subject of begin is also the agent of the argument event, and the latter is either a process or an accomplishment. Equations (23) and (24) show the achieved effects from the derivation of (20).

$$
\text { (a book) }\left(\lambda^{\mathrm{o}} \text { y.begin }{ }_{2}^{\prime} \text { y John }\right):={ }_{\text {forms }} \text { John }+ \text { began }+a+\text { book }
$$


Quantification in frame semantics

$$
\begin{array}{r}
\text { (a book })\left(\lambda^{\mathrm{o}} \text { y.begin }{ }_{2}^{\prime} \text { y John }\right):=_{\text {meaning }} \\
\exists(\downarrow \text { i.book } \wedge \exists(\text { transition } \\
\wedge\langle\text { POST }\rangle((\text { proc } \vee \text { acc }) \wedge\langle\text { AGENT }\rangle(\text { person } \\
\wedge\langle\mathrm{NAME}\rangle \text { John }) \wedge\langle\mathrm{UG}\rangle(\# i))))
\end{array}
$$

It is not specified, though, what kind of event it is: reading, writing, etc. The latter lexically depends on the object. We want to model this dependency by adding lexically determined conditions on the possible models that make the formula true. We already met conditions in the form of meaning postulates, such as (person $\wedge\langle$ NAME $\rangle$ Paul) $\Rightarrow$ man. The conditions we introduce now are different and also make use of another feature of hybrid logic that we have not used so far: actual nominals, and not only state variables. These nominals encode lexical properties of the entities to be used in the meaning representation of the lexical items.

So we introduce the nominals $i_{\text {book }}, i_{\text {reading }}, i_{\text {writing }}, i_{\text {translating }} \ldots$ corresponding to the propositions (or types, in the frame semantics terminology) book, reading, writing, translating... For each of these pairs, the following schema holds:

$$
\left(\langle\text { proto }\rangle i_{p}\right) \Rightarrow p
$$

If we additionally require that each node has a proto attribute, each node in a frame should be associated with a prototypical node named by a nominal, and the proposition that holds at the former can be inferred from the latter.

We also encode that the $i_{\text {book }}$ node is related through the $e-q$ (event quale) relation to some event nominals, requiring the postulates of (26) to hold as well (see Figure 6).

$$
\begin{aligned}
& @_{i_{\text {book }}}\langle e-q\rangle i_{\text {reading }} \\
& @_{i_{\text {book }}}\langle e-q\rangle i_{\text {writing }}
\end{aligned}
$$

Remark (Nominals as prototypical entities). It is very important that the postulates of (26) use nominals rather than properties. Stating these postulates directly with propositions, such as book $\wedge\langle e-q\rangle$ reading, $b o o k \wedge\langle e-q\rangle$ wrting, etc., would amount to require any node where book holds to relate to every (quale) events with an $\langle e-q\rangle$ relation. These 
Figure 6:

Qualia values associated to $i_{\text {book }}$

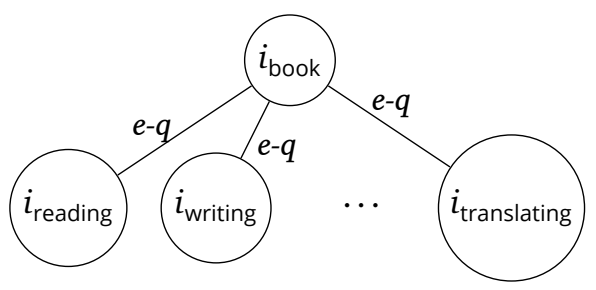

events would then be part of the model even if no linguistic element, such as begin, triggers them.

We can now state the following condition on a node $s$ that has $o$ as undergoer: when looking at the event quale of the prototype of $o$, if we call this event quale $s^{\prime}$, then $s^{\prime}$ should be a prototype of $s$. Formula (27) states this condition in hybrid logic terms. It is the formula used for the interpretations of begin ${ }_{2}$ in Table 8. It can be paraphrased as follows: if $s$ is a state that has $o$ as undergoer, we set $s^{\prime}$ to be a quale event associated to $o$, via a prototype of $o$. For instance, if book holds at $o$, $o \wedge\langle$ proto $\rangle$ is $i_{\text {book }}$. Then $s^{\prime}$ is one of the $i_{\text {reading, }} i_{\text {writing }}$, etc. Say it is $i_{\text {reading }}$. $@_{s}\langle$ proto $\rangle s^{\prime}$ finally ensures that the prototype of $s$ is $i_{\text {reading. Together }}$ with (25), we thus have that reading holds at $s$.

$$
\downarrow s .\langle\mathrm{UG}\rangle\left(o \wedge\langle\text { proto }\rangle\langle e-q\rangle\left(\downarrow s^{\prime} . @_{s}\langle\text { proto }\rangle s^{\prime}\right)\right)
$$

Thus, as Equations (28) and (29) show, together with the postulates (25) and (26), we have the semantic coercion of the object (here a book) to its associated possible telic quales through the prototype relation.

$$
\begin{array}{r}
\text { (a book })\left(\lambda^{\mathrm{o} y} \text {.begin }{ }_{2} y \text { John }\right):=_{\text {forms }} \text { John }+ \text { began }+a+\text { book } \\
(\text { a book })\left(\lambda^{\mathrm{o} y} \text {. begin }{ }_{2} y \text { John }\right):=_{\text {meaning }} \\
\exists(\downarrow \text { i.book } \wedge \exists(\text { transition } \\
\wedge\langle\mathrm{POST}\rangle(\downarrow \text { s. }(\text { proc } \vee \text { acc }) \wedge\langle\text { AGENT }\rangle \text { person } \\
\wedge\langle\mathrm{NAME}\rangle \text { John }) \\
\left.\left.\wedge\langle\mathrm{UG}\rangle\left(\# i \wedge\langle\text { proto }\rangle\langle e-q\rangle\left(\downarrow s^{\prime} . @_{s}\langle\text { proto }\rangle\left(\# s^{\prime}\right)\right)\right)\right)\right)
\end{array}
$$

While accounting for the lexical knowledge, this approach makes no use of a possible specific context where it is not required to use the lexical information. For instance, (30) does not make sense without any context, as ball does not come with a telic quale. 
(30) John began his ball

However, in a context that John was asked to paint a ball, for instance, this information could be used to correctly interpret (30). Such an account could possibly be provided by making use of a selection operator in some context, akin to the one proposed by de Groote (2006) and Lebedeva (2012). The introduction of node binders should indeed allow us to propose such an approach to a continuation-based approach to event context. In particular we may presuppose in the semantics of begin $_{2}$ the path conditions that apply to the object. If this property is already satisfied (for instance by a painting event), nothing else happens beyond the retrieval of this event. Otherwise, the telic quale of the object might be projected, possibly resulting in a failure if no prototypical telic quale is available.

6

\section{GONCLUSION AND PERSPECTIVES}

We used hybrid logic as a means to integrate logical operators with frame semantics. We illustrated the approach with the modelling of quantifier scopes. We embedded the proposed semantic representation within the Abstract Categorial Grammar framework in order to show how to compositionally derive different quantifier scope readings. We also showed how the key ingredients of hybrid logic, nominals, and binders can be used to model semantic coercion, such as the one induced by the begin predicate.

Binding nodes also offers the possibility of using continuation semantics in order to model a dynamic reference to events. In the particular case of semantic coercion, we plan to study how to integrate the model we proposed with a representation of the context. The projection of the telic quale of some (object) entity would then depend on the availability of some previously introduced events.

We also plan to take advantage of the semantic structuring induced by frame semantics to account for representation and copredication of dot type objects. More generally, frame semantics offers several ways to account for subtyping and meaning shifts. A first possibility is to use an ontology by means of axioms (e.g., $\operatorname{man} \Rightarrow$ person). A second possibility is to use structural properties of frames, as proposed for metonymy by Löbner (2014b). A third possibility is to combine the two previous techniques as we propose in this article, encoding 
the qualia structures in prototype frames and linking nodes to their prototypes using axioms.

Finally, we plan to investigate the computational properties of the framework we propose with respect to the hybrid inferential systems (Blackburn and Marx 2002) and the specific properties induced by the frame models we consider, typically the functionality of the attribute relations (Schneider 2007). Modal and hybrid logics indeed generally provide better computability properties than first-order logic in terms of decidability and complexity. Some of these properties are lost when using quantification over nominals (see Section 2.2) and recovering them using restrictions induced by frames on models would be interesting in order to provide semantic representations with tractable automated reasoning capabilities.

\section{REFERENGES}

Carlos Areces, Patrick Blackburn, Antonia Huertas, and María Manzano (2011), Hybrid Type Theory: A Quartet in Four Movements, Principia, 15(2):225-247, DOI: 10.5007/1808-1711.2011v15n2p225.

Carlos Areces, Patrick Blackburn, Antonia Huertas, and María Manzano (2014), Completeness in Hybrid Type Theory, Journal of Philosophical Logic, 43(2-3):209-238, ISSN 0022-3611, DOI: 10.1007/s10992-012-9260-4.

Carlos Areces, Patrick Blackburn, and Maarten Marx (1999), A Road-Map on Complexity for Hybrid Logics, in Jörg FLUM and Mario RODRIGUEZ-ARTALEJO, editors, Computer Science Logic: 13th International Workshop, CSL'99 8th Annual Conference of the EACSL Madrid, Spain, September 20-25, 1999 Proceedings, pp. 307-321, Springer Berlin Heidelberg, DOI: 10.1007/3-540-48168-0_22.

Carlos Areces and Balder ten CATE (2007), Hybrid logics, in Patrick BLACKBURN, Johan Van BENTHEM, and Frank Wolter, editors, Handbook of Modal Logic, volume 3 of Studies in Logic and Practical Reasoning, chapter 14, pp. 821-868, Elsevier, DOI: 10.1016/S1570-2464(07)80017-6.

Jason BALDRIDGE and Geert-Jan KRUIJFF (2002), Coupling CCG and Hybrid Logic Dependency Semantics, in Proceedings of 40th Annual Meeting of the Association for Computational Linguistics, pp. 319-326, Association for Computational Linguistics, Philadelphia, Pennsylvania, USA, DOI: 10.3115/1073083.1073137, ACL anthology: P02-1041.

Hendrik Pieter BARENDREGT (1984), The lambda calculus, volume 103 of Studies in Logic and the Foundations of Mathematics, North-Holland. 
Lawrence BARSALOU (1992), Frames, concepts, and conceptual fields, in Adrienne LEHRER and Eva Feder KITTEY, editors, Frames, fields, and contrasts: New essays in semantic and lexical organization, pp. 21-74, Lawrence Erlbaum Associates, Hillsdale.

Patrick BLACKBuRn (1993), Modal Logic and Attribute Value Structures, in Maarten DE RIJKE, editor, Diamonds and Defaults, volume 229 of Synthese Library, pp. 19-65, Springer Netherlands, ISBN 978-90-481-4286-6, DOI: 10.1007/978-94-015-8242-1_2.

Patrick BLACKBURN and Maarten MARX (2002), Tableaux for Quantified Hybrid Logic, in Uwe EGLY and Chritian G. FERMüLLER, editors, Automated Reasoning with Analytic Tableaux and Related Methods: International Conference, TABLEAUX 2002 Copenhagen, Denmark, July 30 - August 1, 2002 Proceedings, pp. 38-52, Springer, Berlin, Heidelberg, DOI: 10.1007/3-540-45616-3_4.

Patrick BLACKBURN and Maarten De RiJKe (1997), Zooming In, Zooming Out, Journal of Logic, Language and Information, 6(1):5-31, ISSN 0925-8531, DOI: 10.1023/A\%3A1008204403391.

Patrick BLACKBURN and Jerry SELIGMAN (1995), Hybrid languages, Journal of Logic, Language and Information, 4(3):251-272, DOI: 10.1007/BF01049415. Johan Bos (1995), Predicate Logic Unplugged, in Proceedings of the Tenth Amsterdam Colloquium, ht tp ://www . let . rug.nl/bos/pubs/Bos1996AmCo.pdf.

Haskell Brooks CURRY (1961), Some Logical Aspects of Grammatical Structure, in Roman JAKOBSON, editor, Structure of Language and its Mathematical Aspects: Proceedings of the Twelfth Symposium in Applied Mathematics, pp. 56-68, American Mathematical Society.

Philippe DE GROOTE (2001), Towards Abstract Categorial Grammars, in Association for Computational Linguistics, 39th Annual Meeting and 10th Conference of the European Chapter, Proceedings of the Conference, pp. 148-155, ACL anthology: P01-1033.

Philippe DE GROOTE (2002), Tree-Adjoining Grammars as Abstract Categorial Grammars, in Proceedings of the Sixth International Workshop on Tree Adjoining Grammars and Related Frameworks (TAG+6), pp. 145-150, Università di Venezia, http://www. loria.fr/equipes/calligramme/acg/ publications/2002-tag+6. pdf.

Philippe DE GROOTE (2006), Towards a Montagovian account of dynamics, in Masayuki GIBSON and Jonathan HOWELL, editors, Proceedings of Semantics and Linguistic Theory (SALT) 16, DOI: 10.3765/salt.v16i0.2952.

Philippe DE GRoote and Sylvain Pogodalla (2004), On the expressive power of Abstract Categorial Grammars: Representing context-free formalisms, Journal of Logic, Language and Information, 13(4):421-438, DOI:

10.1007/s10849-004-2114-x, HAL open archive: inria-00112956. 
Charles J. Fillmore (1977), The case for case reopened, in Peter ColE and Jerrold M. SADOCK, editors, Grammatical Relations, volume 8 of Syntax and Semantics, pp. 59-81, Academic Press, New York.

Massimo FRANCESCHET and Maarten DE RIJKE (2006), Model checking hybrid logics (with an application to semistructured data), Journal of Applied Logic, 4(3):279-304, DOI: 10.1016/j.jal.2005.06.010.

Daniel GALlin (1975), Intensional and Higher-Order Modal Logic, North-Holland. Thomas GAMERSChlaG, Doris Gerland, Rainer OsswAlD, and Wiebke Petersen, editors (2014), Frames and Concept Types, volume 94 of Studies in Linguistics and Philosophy, Springer International Publishing, DOI:

10.1007/978-3-319-01541-5.

Seohyun IM and Chunngmin LEE (2015), A Developed Analysis of Type Coercion Based on Type Theory and Conventionality, in Robin COOPER and Christian RETORÉ, editors, Type Theory and Lexical Semantics, ESSLLI 2015, Barcelona, Spain, http ://www. lirmm.fr/tytles/Articles/Im . pdf.

Laura KALLMEYER and Rainer OsswALD (2013), Syntax-Driven Semantic Frame Composition in Lexicalized Tree Adjoining Grammars, Journal of Language Modelling, 1(2):267-330, DOI: 10.15398/jlm.v1i2.61.

Laura KALLMEYER, Rainer OssWALD, and Sylvain PogodAlla (2015), Progression and Iteration in Event Semantics - An LTAG Analysis Using Hybrid Logic and Frame Semantics, in Colloque de Syntaxe et Sémantique à Paris (CSSP 2015), HAL open archive: hal-01184872.

Laura KALLMEYER and Frank RICHTER (2014), Quantifiers in Frame Semantics, in Glyn MORRILL, Reinhard Muskens, Rainer OsswALD, and Frank RICHTER, editors, Formal Grammar, volume 8612 of Lecture Notes in Computer Science, pp. 69-85, Springer, DOI: 10.1007/978-3-662-44121-3_5.

Laura KALLMEYER and Maribel RoMERo (2008), Scope and Situation Binding for LTAG, Research on Language and Computation, 6(1):3-52, DOI:

10.1007/s11168-008-9046-6.

Joachim LAMBEK (1958), The Mathematics of Sentence Structure, American Mathematical Monthly, 65(3):154-170.

Ekaterina LEBEDEVA (2012), Expression de la dynamique du discours à l'aide de continuations, Ph.D. thesis, Université de Lorraine, in English.

Sebastian LÖBNER (2014a), Evidence for frames from human language, in Gamerschlag et al. (2014), chapter 2, pp. 23-67, DOI:

10.1007/978-3-319-01541-5_2.

Sebastian LÖBNER (2014b), Frames and metonymy - Shifting the center and refocusing the frame, Concept Types and Frames in Language, Cognition, and Science (CTF14), invited talk, http: //Www.sfb991. uni-duesseldorf.de/ fileadmin/Vhosts/SFB991/CTF14Abstr/Loebner_-_AK.pdf.

Igor A. MEL'ČUK, André CLAS, and Alain POLGUÈRE (1995), Introduction à la lexicologie explicative et combinatoire, Éditions Duculot, Louvain-la-Neuve. 
Marc Moens and Mark STEedman (1988), Temporal Ontology and Temporal Reference, Computational Linguistics, 14(2):15-28, ACL anthology: J88-2003. Richard Montague (1974), The Proper Treatment of Quantification in Ordinary English, in Formal Philosophy: Selected Papers of Richard Montague, Yale University Press, re-edited in "Formal Semantics: The Essential Readings", Paul Portner and Barbara H. Partee, editors. Blackwell Publishers, 2002.

Reinhard Muskens (2013), Data Semantics and Linguistic Semantics, in Maria AlOni, Michael Franke, and Floris RoElofsen, editors, The dynamic, inquisitive, and visionary life of $\phi, ? \phi$, and $\diamond \phi$, chapter 24, pp. 175-183, Pumbo.nl, http://WWW.illc.uva.nl/Festschrift-JMF/papers/23_Muskens.pdf. Rainer OsSWALD and Robert D. VAN VALIN, Jr. (2014), FrameNet, Frame Structure, and the Syntax-Semantics Interface, in Gamerschlag et al. (2014), chapter 6, pp. 125-156, DOI: 10.1007/978-3-319-01541-5_6.

Wiebke Petersen (2007), Representation of Concepts as Frames, The Baltic International Yearbook of Cognition, Logic and Communication, 2:151-170, http://user.phil-fak. uni-duesseldorf.de/ petersen/paper/ Petersen2007_proof.pdf.

Sylvain Pogodalla (2004), Computing Semantic Representation: Towards ACG Abstract Terms as Derivation Trees, in Seventh International Workshop on Tree Adjoining Grammar and Related Formalisms - TAG + 7, pp. 64-71, Vancouver, BC, Canada, HAL open archive: inria-00107768.

Alain PolguÈre (2003), Lexicologie et sémantique lexicale, Les Presses de l'Université de Montréal.

James Pustejovsky (1998), The Generative Lexicon, MIT Press.

James Pustejovsky and Pierrette Boulllon (1995), Aspectual Coercion and Logical Polysemy, Journal of Semantics, 12(2):133-162, DOI:

10.1093/jos/12.2.133.

Thomas SCHNEIDER (2007), The Complexity of Hybrid Logics over Restricted Classes of Frames, Ph.D. thesis, University of Jena, Germany, http://www.cs.man.ac.uk/ schneidt/publ/sch07_phd.pdf.

Balder TEN CATE and Massimo FRANCESCHET (2005), On the Complexity of Hybrid Logics with Binders, in Luke ONG, editor, Computer Science Logic: 19th International Workshop, CSL 2005, 14th Annual Conference of the EACSL, Oxford, UK, August 22-25, 2005. Proceedings, pp. 339-354, Springer Berlin Heidelberg, DOI: $10.1007 / 11538363 \_24$.

This work is licensed under the Creative Commons Attribution 3.0 Unported License. http://creativecommons .org/licenses/by/3.๑/

(c) BY 